\title{
A Parallel Randomised Controlled Trial of Single Dose Vitamin D3 Versus Daily Supplementation with Pentavite in Infants with Vitamin D Deficiency
}

\author{
Simon A Costello ${ }^{1 *}$, and Margaret Staples ${ }^{2}$ \\ ${ }^{1}$ Department of Paediatrics, Monash University, Clayton Vic, Australia \\ ${ }^{2}$ Department of Clinical Epidemiology Cabrini Institute, Malvern Vic, Australia
}

${ }^{\star}$ Correspondence to: Simon A Costello, Department of Paediatrics, Monash University, Clayton Vic 3168, Australia; Tel: 9509 8688; E-mail: costellosimon@bigpond. com

Received: May 14, 2018; Accepted: May 24, 2018; Published: May 25, 2018;

\begin{abstract}
The main aim for the trial involving infants of mothers with Vitamin D Deficiency was to compare two treatment modalities that being, A single dose of Vitamin D3 of 50,000 units vs a daily dose of 400 units for 4 weeks. In addition, the secondary finding was that incidence of true Vitamin D deficiency in the infants born to mothers with Vitamin D deficiency. We were able to demonstrate that a significant percentage of infants born to mothers with Vitamin D deficiency had been treated with Vitamin D during the pregnancy did in fact have more Vitamin D levels in the normal range. As a result, this reduced the number of potential infants that were able to be enrolled in the study. We were able to identify 93 infants with Vitamin D deficiency using the definition of a level Vitamin D to a less than $75 \mathrm{nmol}$ per litre. The mean Vitamin D level at birth in the Pentavite group was slightly higher than the Vitamin D3 group.
\end{abstract}

Keywords: Vitamin D deficiency; hypocalcaemia; Pentavite; Caucasian and non-Caucasian infants

\section{Introduction}

Vitamin $\mathrm{D}$ has been progressively linked to a variety of disease conditions in children over the last decade. Its role in bone mineralisation has long been recognised. More recently Vitamin D deficiency has been associated with increased prevalence of allergic reactions [1]. It has also been reported that higher vitamin D intake offers protection against episodes of wheeze in children under 5 Years [2].

In addition, a strong correlation has been reported between Vitamin D deficiency and cardiovascular disease [3]. Furthermore, the risk of developing type 1 diabetes and systemic lupus erythematosis has been linked with low early intake of Vitamin D $[4,5]$. The importance of Vitamin D supplementation in preterm infants is well documented. Its role in prevention of hypocalcaemia during rapid bone growth is of primary importance as well as prevention of reduced bone mineralisation with potential for rickets. It is reported that sun exposure of the body up to 15 minutes in lightly pigmented adults may generate up to 20,000 units of Vitamin D3 over 24 hours whereas those with darker pigmentation may require 5 - 10 times the exposure for a similar result $[6,7]$.

Sun avoidance for infants may compromise Vitamin D levels. In addition, breast milk has very low levels of Vitamin D. The place of Vitamin D supplementation in term infants is less clear. The issue has risen in prominence with recognition of the significant percentage of pregnant women who have been found to be Vitamin D deficient [8].
In a Cochrane review [9] in 2002 it was noted that data regarding ideal Vitamin D requirements in pregnancy are limited. In addition, women with darker skin would likely need additional Vitamin D supplements.

An association between umbilical cord $25-\mathrm{OH}-\mathrm{D}$ levels and head circumference at 3 and 5 months postnatal age has also been reported [10]. Another study reported increased bone mineralisation in children at 9 years where higher maternal Vitamin D levels were recorded during pregnancy [11]. Infants of African- American mothers who are breast fed only without formula supplements are at greater risk of Vitamin D deficiency for reasons previously stated [12].

In their article Jain et al., [13] report Vitamin D deficiency and insufficiency in healthy term breast fed 3-month-old infants and their mothers. There is however lack of consensus on a definition of true Vitamin D insufficiency. They recommend maternal supplements, increased sun exposure for the mother and possibly routine supplementation for infants.

Wagner et al., [14] report significant and prolonged increases in Vitamin D levels through to 7 months in infants given an oral Vitamin D3 supplement.

It has become an accepted practice among many paediatricians to give a single dose of 50,000 units of Vitamin D 3 to infants of Vitamin D deficient mothers. The evidence for this practice is not well established. In addition, there is the possibility that a large dose of Vitamin D3 given to an infant who has an already high Vitamin D level could lead to acute Vitamin D intoxication. 
This study aimed to;

1. determine the incidence of Vitamin D deficiency in babies born to mother with low Vitamin D levels detected in pregnancy who were on supplemental Vitamin D, and,

2. compare mean serum Vitamin D levels at 6 weeks after a single dose Vitamin D3 50,000 units with those of oral Pentavite (400 u Vit D) given daily for 6 weeks.

\section{Methods}

We performed a randomised controlled trial in newborn infants of Vitamin D deficient mothers to compare a single 50,000 unit dose of Vitamin D3 (Single dose group) with daily Pentavite (daily dose group). Infants were enrolled from August 2012 to October 2014.

Women who had been identified at about 12 weeks gestation with vitamin D levels $<75 \mathrm{nmol} / \mathrm{L}$ were selected. All were prescribed vitamin D supplements. At the birth of their infant ( $>36$ weeks) informed consent was obtained to determine the infant's vitamin D levels using either cord blood or the Newborn Screening Test.

Infants with levels in the normal range were noted and infants with levels less than $75 \mathrm{mmol} / \mathrm{s}$ were randomised by sealed envelopes to receive either the single dose group or the daily dose group. Repeat Vitamin D levels were then obtained from each group at 6 weeks.

Demographic and clinical data were collected on maternal age, sex of neonate, ethnic background, evidence of vomiting of bolus vit D, questioning for compliance with daily vit D supplementation, use of vitamin D supplementation by single bolus group.

\section{Sample size and statistical analysis}

The primary outcome measures were the proportion in each group attaining normal vitamin $\mathrm{D}$ levels $(>75 \mathrm{nMol} / \mathrm{L})$ at 6 weeks and the mean change per group in vitamin D level between day 3 and 6 weeks. It was estimated that 40 infants per group would have $80 \%$ power at the $5 \%$ significance level to show a difference of at least $20 \%$ between the two groups in the proportion achieving normal Vitamin D levels at 6 weeks.

We performed an intention to treat analysis. Mean changes in vitamin D levels between groups were compared using t-tests and logistic regression was used to determine associations between successful outcome (Vitamin D reaching normal levels) and clinicdemographic variables.

\section{Results}

The flow diagram for study recruitment is shown in Figure 1. A total of 267 Vitamin D deficient mothers were treated during their pregnancy. Table 1 shows the ethnicity and Vitamin D status at birth of the infants of these mothers. There was no significant difference in the proportion of Caucasian and non-Caucasian infants with Vitamin $\mathrm{D} \leq 75 \mathrm{nmol} / \mathrm{L}$ at birth $(\mathrm{p}=0.37)$.

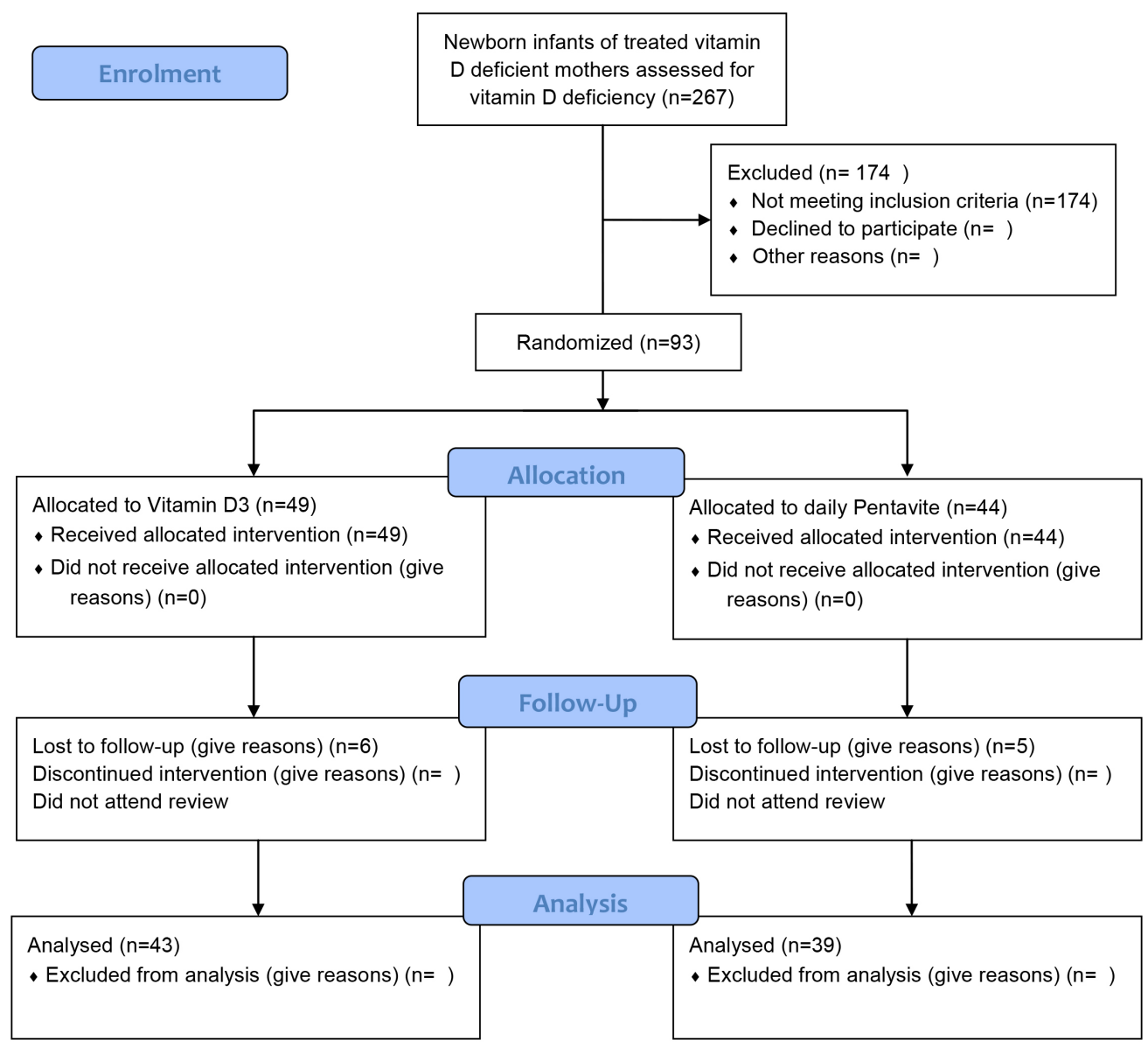


The overall incidence of Vitamin D deficiency in the two treatment groups of non-Caucasian background was 39 out of 87 i.e, $44.8 \%$. In the Caucasian population the incidence was 54 out of 180 i.e, $30 \%$. The 93 infants with low Vitamin D levels were randomised to treatment groups - 49 to the single dose group and 44 to the daily dose group. The baseline characteristics of the two groups is shown in Table 1 . The mean vitamin D level at birth was higher in the Pentavite group (Figure 2).

Table 1a. Ethnicity of infants of Vitamin D deficient mothers $(n=267)$.

\begin{tabular}{|l|c|c|c|}
\hline & Caucasian & Non-Caucasian & Total \\
\hline Vitamin D $>75 \mathrm{nmol} / \mathrm{L} 1$ & $126(65.1 \%)$ & $48(69.6 \%)$ & 174 \\
\hline Vitamin $\mathrm{D} \leq 75 \mathrm{nmol} / \mathrm{L}$ & $54(36.4 \%)$ & $39(30.4 \%)$ & $93(34.8 \%)$ \\
\hline Total & 180 & 87 & 267 \\
\hline
\end{tabular}

Table 1b. Baseline characteristics of each group.

\begin{tabular}{|l|c|c|}
\hline Characteristic & $\begin{array}{c}\text { Single dose Vitamin } \\
\text { D3 group }(\mathbf{n = 4 9 )}\end{array}$ & $\begin{array}{c}\text { Pentavite group } \\
(\mathbf{n}=\mathbf{4 4})\end{array}$ \\
\hline Male / Female & $24 / 25$ & $21 / 23$ \\
\hline Caucasian/Non-Caucasian & $26 / 23$ & $28 / 16$ \\
\hline $\begin{array}{l}\text { Mean (SD) vitamin D level } \\
\text { (nmol/L) }\end{array}$ & $50.40(13.33)$ & $57.41(11.88)$ \\
\hline SD: Standard Deviation & & \\
\hline
\end{tabular}

Seven infants in the single dose group and 5 in the daily dose group did not attend for the 6 weeks review and were lost to follow-up. At 6 weeks 72 of the 81 infants (88.9\%) had achieved normal vitamin D levels (Table 2). The single dose Vitamin D3 group were more likely to have achieve normal levels that the Pentavite group (97.6\% single dose group vs $79.5 \%$ Pentavite group) (OR 10.58; 95\% confidence interval (CI) $1.26,89.08$ ) (Figure 3 ). The single dose group also showed a greater mean change from birth to 6 weeks (Figure 4).

\section{Vitamin $D$ levels at birth by group}
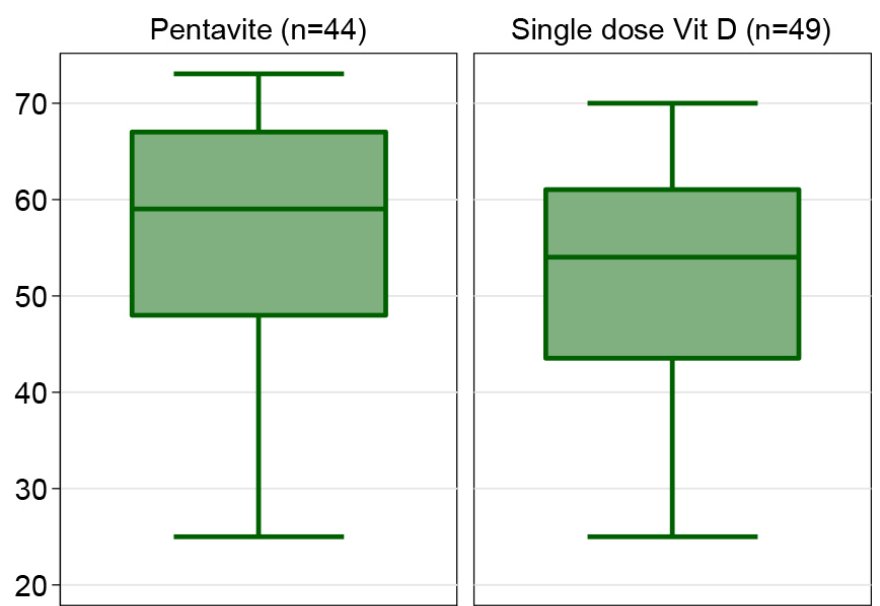

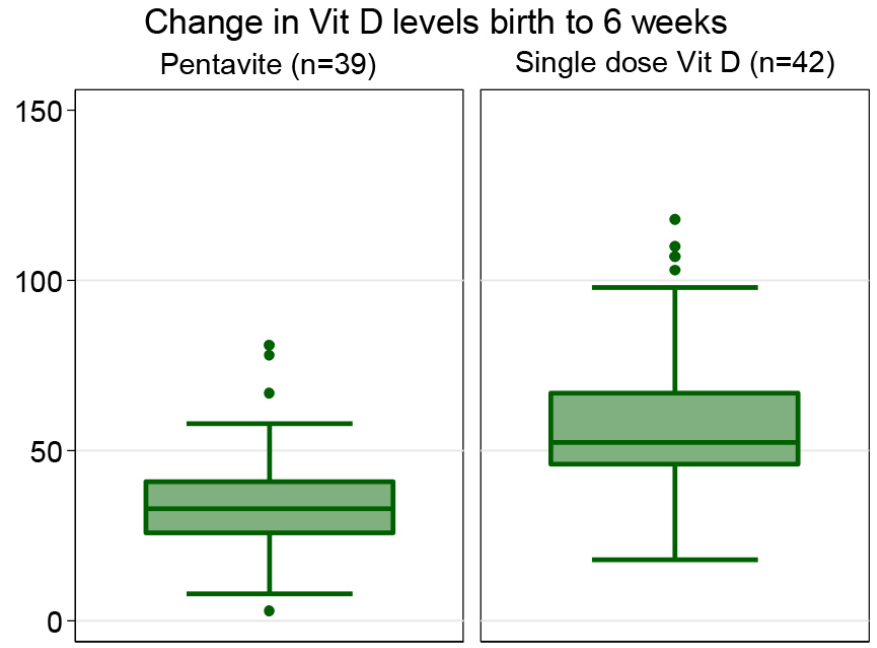

Figure 3. Change in Vit D levels birth to 6 weeks.

Mean Vit D levels $\pm S D$

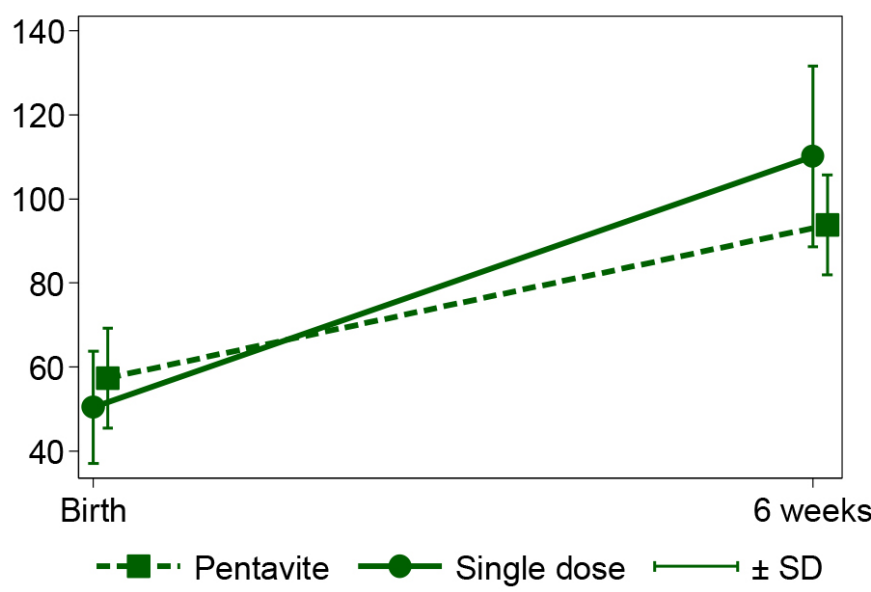

Figure 4. Mean Vit D levels \pm SD.

Table 2. Results by group.

\begin{tabular}{|l|c|c|}
\hline Six weeks outcomes & $\begin{array}{c}\text { Single dose Vitamin } \\
\text { D3 group (n=42) }\end{array}$ & $\begin{array}{c}\text { Pentavite group } \\
(\mathbf{n = 3 9 )}\end{array}$ \\
\hline Percent achieving normal levels & 97.6 & 79.5 \\
\hline $\begin{array}{l}\text { Mean (SD) level at 6 weeks } \\
(\mathrm{nmol} / \mathrm{L})\end{array}$ & $110.10(21.44)$ & $93.79(21.33)$ \\
\hline $\begin{array}{l}\text { Mean (SD) change from birth } \\
\text { (nmol/L) }\end{array}$ & $59.69(23.94)$ & $36.38(17.62)$ \\
\hline SD: Standard Deviation & & \\
\hline
\end{tabular}

All infants given a bolus dose tolerated it; no vomiting was reported. Supplemental Vit D administration was not reported by mothers at the 6-week review.

Figure 2. Vitamin D levels at birth by group. 


\section{Conclusion}

In conclusion we recommend the following management for women identified as Vitamin D deficient during pregnancy;

1. Vitamin D supplementation during pregnancy.

2. Obtain a Vitamin D level on the infant soon after delivery.

3. Give 50,000 of Vitamin D3 orally as a single dose which will ensure adequate Vitamin D levels are achieved through to at least 6 weeks of age.

\section{Acknowledgement}

Ethics approval for this study was obtained from the Cabrini Human Research Ethics Committee.

\section{References}

1. Litonjua AA, Weiss ST (2007) Is vitamin D deficiency to blame for the asthma epidemic? J Allergy Clin Immunol 120: 1031-1035. [Crossref]

2. Camargo CA Jr, Rifas-Shiman SL, Litonjua AA, Rich-Edwards JW, Weiss ST, et al. (2007) Material intake of vitamin D during pregnancy and risk of recurrent wheeze in children at 3 years of age. Am J Clin Nutr 13(3): 788-795. [Crossref]

3. Kendrick J, Targher G, Smits G, Chonchol M (2009) 25-Hydroxyvitamin D deficiency is independently associated with cardiovascular disease in the Third National Health and Nutrition Examination Survey. Atherosclerosis. 205: 255-260. [Crossref]

4. Hyppönen E, Läärä E, Reunanen A, Järvelin MR, Virtanen SM (2001) Intake of vitamin D and risk of type 1 diabetes: a birth-cohort study. Lancet 358: 1500-1503. [Crossref]

5. Kamen DL, Cooper GS, Bouali H, Shaftman SR, Hollis BW, et al. (2006) Vitamin D deficiency in systemic lupus erythematosus. Autoimmun Rev 5: 114-117. [Crossref]

6. Matsuoka LY, Wortsman J, Haddad JG, Kolm P, Hollis BW (1991) Racial pigmentation and the cutaneous synthesis of vitamin D. Arch Dermatol 127: 536538. [Crossref]

7. Matsuoka LY, Wortsman J, Hollis BW (1990) Suntanning and cutaneous synthesis of vitamin D3. J Lab Clin Med 116: 87-90. [Crossref]

8. Bischoff-Ferrari HA1 (2011) Vitamin D: role in pregnancy and early childhood. Ann Nutr Metab 59: 17-21. [Crossref]

9. Mohamed K, Gulmezoglu AM (2002) Vitamin D supplementation in pregnancy. The Cochrane Library. Oxford, United Kingdom. Update software.

10. Brooke OG, Butters F, Wood C (1981) Intrauterine vitamin D nutrition and postnatal growth in Asian infants. Br Med J (Clin Res Ed) 283: 1024. [Crossref]

11. Javaid MK, Crozier SR, Harvey NC, Gale CR, Dennison EM, et al. (2006) Maternal vitamin D status during pregnancy and childhood bone mass at age 9 years: a longitudinal study. Lancet 367: 36-43. [Crossref]

12. Kreiter SR, Schwartz RP, Kirkman HN Jr, Charlton PA, Calikoglu AS, et al. (2000) Nutritional rickets in African American breast-fed infants. J Pediatr 137: 153-157. [Crossref]

13. Jain V, Gupta N, Kalaivani M, Jain A, Sinha A, et al. (2011) Vitamin D deficiency in healthy breastfed term infants at 3 months \& their mothers in India: seasonal variation \& determinants. Indian J Med Res 133: 267-273. [Crossref]

14. Wagner CL, Howard C, Hulsey TC, Lawrence RA, Taylor SN, et al. (2010) Circulating 25-hydroxyvitamin d levels in fully breastfed infants on oral vitamin d supplementation. Int J Endocrinol 2010: 235035. [Crossref]

\section{Citation:}

Simon A Costello and Margaret Staples (2018) A Parallel Randomised Controlled Trial of Single Dose Vitamin D3 Versus Daily Supplementation with Pentavite in Infants with Vitamin D Deficiency. Internal Med Res Open J Volume 3(3): 1-4. DOI: 10.31038/IMROJ.1000126 